\title{
30) CHEMOTHERAPY OF FILARIASIS
}

$\mathrm{W}$ ORKERS engaged in the search of a cure for filarioid infestatons in man and animals will welcome the paflicution* of a symposium of fifteen papers by thir yeight contributors who attended a conference pr "The Chemotherapy of Filariasis" unde Nthe auspices of the Section of Biology of the New York Academy of Sciences on October 17 and 18, 14. T. The term 'filariasis' in this publication signifies an infestation with a member of the nematode super-family Filarioidea. The use of this term recalls the days when the nematodes recovered from the lymphatic, eirculatory or connective tissues or from the serous cavities of man and the lower vertebrates were all grouped in the genus Filaria, Mueller, 1787. Purists in medical and veterinary sciences, adopting the present-day classifications of taxonomy in biology, may maintain rightly that the term 'filariasis' should no longer be employed to indicate all infestations due to members of the genera Onchocerca, Loa, Wuchereria, Acanthocheilonema, Mansonella, Dirofilaria, etc. The use of the terms 'onchocerciasis', 'loaiasis', etc., indicates specifically the genera involved.

L. T. Coggeshall deals briefly with the history of filariasis due to $W$. bancrofti in the United States, and discusses symptomatology, pathology, immunology, prophylaxis and control. He includes remarks on loaiasis, onchocerciasis and dracontiasis. Filarioid infestations are, as a rule, chronic in character. Clinical manifestations may not become evident for years. There is still controversy as to whether the induration of the tissues in cases of elephantiasis, induced by $W$. bancrofti and occasionally by $O$. volvulus, is not due to secondary bacterial invasion. Coggeshall observes that the pathological changes are not typical of streptococcal infections and that they do not respond to agents which have a specific effect on the streptococcus.

No marked degree of immunity against further re-infection has been detected in individuals already infested, and cases of the same individual being infested with two or more members of the superfamily are not uncommon. Coggeshall estimates that up to two million human beings are infested with worms of this group, while other American workers claim that the victims number twenty million. The incidence in domestic animals (horse, ox, sheep, goat, pig, camel, dog, cat, etc.) is high and widespread even in temperate climates. There is no doubt that the efficiency, productivity and welfare of many countries in the tropics and subtropics are seriously impaired by filarioid infestations in man and his livestock.

The low mortality associated with these forms of helminthiasis has rendered the general public indifferent to the sufferings of the afflicted. In the endemic regions, the efforts of the public health authorities to control and eradicate these maladies by prophylactic measures are handicapped by many difficulties, and Coggeshall hopes that the discovery of an effective chemotherapeutic agent would facilitate the elimination of infections from the endemic areas.

The general experimental methods used in studying 'filaricides' are discussed by R. I. Hewitt. The cotton rat (Sigmodon hispidus), naturally infected with a

" "The Chemotherapy of Filariasis", by various contributors. Vol. 50, art. 2. pp. 19-170 (Ann. N.Y. Acad. Sci., 1948). filariid, Litomosoides carinii, has been found a most suitable experimental animal for testing the efficiency of 'filaricides'. Dogs, infected with the blood filariid, Dirofilaria immitis, are used for comparative and confirmative work; but they are considered too large for use in ordinary routine work. The estimation of the efficiency of a 'filaricide' against the species parasitizing the cotton rat is based on destruction of the microfilariæ and its action on the adult worms. Hewitt attributes the disappearance of the micro. filariæ in infected cotton rats to the death of the adult worms and not to a direct action upon the microfilariæ. He experimented with various piperazine compounds and admits that the use of controlled quantitative infestations has many advantages over naturally established infections; he considers that the relationship between drug dosage and the intensity of infestation can be gauged best in standardized infections. He notes that the filariids of the cotton rat, dog and man belong to different genera and that the different worms may respond differently to the same drug; but he believes that the utilization of infected cotton rats for testing the filaricidal efficiency of drugs may facilitate the discovery of a 'cure' in man.

G. F. Otto and T. H. Maren discuss the use of arsenicals in filarioid infestations. The literature and their own investigations indicate that the trivalent organic arsenicals are active in both human and canine infestations. Arsenamide kills $D$. immitis in dogs and, in well-tolerated doses, has been found to destroy the microfilariæ of $W$. bancrofti. They add : "It remains to be determined whether or not the adults of $W$. bancrofti are killed by this treatment". H. W. Brown reports on the treatment of filariid infestations with anthiomaline in the dog, cotton rat and man, and records that this compound reduced permanently the microfilariæ counts in individuals infested with $W$. bancrofti. The necessity for repeated injections, and also its toxicity, limit its usefulness, and Brown considers it only 'a stepping-stone' to be replaced by an orally administered non-toxic remedy. James T. Culbertson concludes: "Wuchereria bancroft can bo eradicated from many patients by the inten. sive administration of any of the several compounds of antimony and arsenic". He records that the pentavalent antimonial, 'Neostibosan', is well tolerated by man and has a marked destructive effect on the worms.

Twenty-five volunteers suffering from onchocerciasis were treated by F. C. Barther, T. A. Burch, D. B. Cowie, L. L. Ashburn and F. J. Brady with trivalent antimony compounds ('Stibophen' and tartar emetic). They observed no decrease in the number of microfilariæ or loss of motility in the adult worms. In one case, the administration of tartar emetic, prepared from radioactive antimony, showed that antimony had been taken up by the worm in one nodule. Harry M. Rose deals with the tolerance of antimony and arsenic compounds in intensively treated patients. The drugs used were: 'Neostibosan', 'Neostam', 'Fuadin', 'Anthiomaline' (Specia), 'An. thiomaline' (Merck), urea, stebamine, tartar emetic and stebanose.

Ernest Bueding reports that cyanine dyes in con. centrations as low as one in forty millions inhibit the oxidative metabolism of $L$. carinii. The lethal action 
of syanines in the case of $L$. carinii infestations is attributed to inhibition of the respiratory metabolism. He observes that, in contrast to vertebrates, the metabolic characteristics of invertebrates may vary greatly from species to species, and that morphologically related parasites need not necessarily be sensitive to the same chemotherapeutic agents. He rightly observes that investigations of the biochemical characteristics of pathogenic parasites may lead to the replacement of the present empirical methods by more rational methods of treatment. Lawrence Peters records that certain cyanine dyes had no chemotherapeutic effect on $W$. bancrofti although they proved lethal to $L$. carinii. $\mathrm{He}_{\mathrm{e}}$ believes that this must be attributed, not to the failure of the drug to reach $W$. bancrofti, but to a difference in the meta. bolic characters of the two species.

S. Kushner, L. M. Brancone, Hewitt, W. L. McEwen, Y. SubbaRow, H. W. Stewart, R. J. Turner and J. J. Denton discuss the preparation of the piperazine compounds used in the search for an active filaricide which can be administered orally and is non-metallic. Hewitt, D. E. White, Kushner, W. S. Wallace, Stewart and SubbaRow report on the 'filaricidal' properties of various piperazine compounds, particularly 'Hetrazan', in dogs and cotton rats. Several of the compounds produced immediate and sharp reductions in microfilaria counts in infected cotton rats when administered orally or intraperitoneally; sometimes relapses occurred. 'Hetrazan' was also lethal to the adult worms in dogs and cotton rats. These workers record that they failed to find dead worms at autopsy in some of the treated dogs, and it seems possible that these dogs were infested not with $D$. immitis but with D. acutiuscula, which occur in the subcutis and have been reported from the Canadian lynx (Lynx canadensis). They examined only the heart and lungs for dead worms. B. K. Hamed, R. W. Cunningham, Sybella Halliday, R. E. Vessey, N. N. Yuda, Mary C. Clark and SubbaRow give the structural formula of 'Hetrazan', report on its toxicity for mice, rats, guinea pigs, rabbits, cats and dogs, and cite some of its pharmacological properties.

D. Santiago-Stevenson, J. Oliver-Gonzalez and Hewitt report on the treatment with 'Hetrazan', taken by the mouth, of twenty-six individuals infected with $W$. bancrofti. The dosage varied from $0.5 \mathrm{mgm}$. to $2 \mathrm{mgm}$. per $\mathrm{kgm}$. of body-weight thrice daily for periods varying from three to twenty-two days. For optimum results they suggest a dosage of $2 \mathrm{mgm}$. per $\mathrm{kgm}$. body-weight thrice daily for a period of two to three weeks. There was a rapid disappearance of microfilariæ from the blood, and the development of nodular swellings at certain points of the body, usually infested by the adult worms, is accepted as proof that the compound killed some of the adult worms.

A study of "The Chemotherapy of Filariesis" will reveal that the search for a cure for filarioid infestations in man and his stock cannot be conducted bebind 'iron curtains'. 'The workers in the various fields of science must co-operate and collaborate more and more closely than is usually the case. A better knowledge of the chemical composition and the metabolism of the various helminths parasitizing man, animals and plants may contribute materially towards the discovery of 'cures' which will not only relieve, to some degree, the present world-wide shortages of food, but will also restore normal health to many all over the world.

\section{SOIL THERMODYNAMICS AND ROAD ENGINEERING}

\author{
By J. D. COLEMAN
}

Road Research Laboratory, Harmondsworth, Middlesex

$T$ $\mathrm{HE}$ movement of moisture in soil is of importance to the civil engineer owing to the effect which changes in moistyre content may have on the strength and the volupe of the soil. In the case of roads, both the stability and the riding qualities of the surface gre involved.

The hermodynamics of the moisture contained in hygroscopic materials such as paper, tobacco and textiles has received considerable attention, and much has already been done, particularly by agricultural scientists, in applying this work to soils. A fundamental approach on these lines to the many problems associated with the transfer of moisture in road foundations is at present being made at the Road Research Laboratory.

The potential causing the migration of soil water may be regarded either as a hydraulic pressure or a vapour pressure gradient. Movements cease only when suction and vapour pressure conditions are in equilibrium with the acting gravitational field under the prevailing temperature conditions.

The curvature of each of the air/water interfaces in the soil pores is a function of the pressure change through the meniscus and of the relative humidity at the interface. The relationship between relative humidity is given to a close degree of approximation by the well-knorm Kelvin equation, which may be written,

$$
\frac{\rho \cdot R \cdot \theta}{M} \cdot \log _{e} \frac{H}{100}=-\frac{2 T}{r} .
$$

The actual change in pressure through the meniscus, p.g.h $=2 T / r$, can be expressed in terms of Schofield's $p F^{\prime}$ function,

$$
p F=\log _{10} h=\log _{10} \frac{2 T}{\rho \cdot r \cdot g} .
$$

Equations (1) and (2) have been used by Schofield and others in the determination of the $p F$ of soils at low moisture contents.

In the soil itself, the curvature of the air/water interfaces depends on the moisture content and on certain characteristics of the soil solids, the chief of which appears to be the particle-size distribution. It follows that the suction/moisture content and the relative humidity/moisture content relationships depend on soil type; and, in fact, the moisture contents of two dissimilar soils in moisture equilibrium may be greatly different. In road construction, where it is common on clay soils to use a stable sandy soil to form part of the road foundation, the suction/moisture content relationships are useful in estimating the equilibrium moisture (and strength) conditions.

Road foundations are subjected to both the daily and the yearly temperature cycles. It is therefore necessary to ascertain the effect of temperature on the suction and humidity relationships. The variation of the density of the soil water with temperature causes only a second-order variation in the radii of the air/water interfaces. Neglecting this variation, it follows from (2) that at two temperatures $\theta_{1}$ and $\theta_{2}$,

$p F_{\theta_{1}}-p F_{\theta_{2}}=$

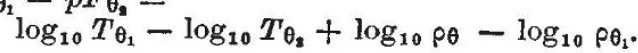

